\title{
4 Andrologie in der Klinik
}

\author{
Gerhard Haidl, Hans-Christian Schuppe, Wolfgang Weidner und \\ Uwe Paasch
}

Die Aufgabengebiete der Andrologie umfassen wie in Kapitel IV.3 dargestellt die Diagnostik und Therapie männlicher Fertilitätsstörungen, Erektions- und Sexualstörungen, Krankheiten des Hodens (Hodentumoren) und der Prostata (Prostatitis, benignes Prostatasyndrom, Prostata-Karzinom), Störungen der endokrinen Hodenfunktion (primärer, sekundärer Hypogonadismus) sowie der Pubertätsentwicklung, die Thematik des alternden Mannes und der Hormonersatztherapie und Krankheitsbilder der männlichen Brustdrüse. Ebenso gehören Fragen der Prävention im Hinblick auf Arzneimittelnebenwirkungen und andere exogene Noxen, der Fertilitätsprophylaxe (Kryokonservierung von Spermien z.B. vor potenziell gonadotoxischen Therapiemaßnahmen bei onkologischen Erkrankungen), der Familienplanung und Kontrazeption aufseiten des Mannes sowie forensische Vaterschaftsprobleme zur Andrologie in Klinik und Praxis. In andrologischen Zentren spielen darüber hinaus klinische und grundlagenorientierte Forschung eine wesentliche Rolle.

Eine andrologische Abteilung bzw. Funktionseinheit an einer (Universitäts-)Klinik sollte über die volle Weiterbildungsermächtigung für das Teilgebiet der Andrologie (18 Monate) verfügen und damit das gesamte Spektrum der diagnostischen und therapeutischen Möglichkeiten anbieten können. Darüber hinaus ist die Andrologie ein ausgesprochen interdisziplinär ausgerichtetes Fach: Im Rahmen der Betreuung von Paaren mit unerfülltem Kinderwunsch ist insbesondere eine enge Kooperation mit der Gynäkologie/ Reproduktionsmedizin erforderlich, weiterhin sind verschiedene Disziplinen wie z.B. Humangenetik, Pädiatrie, Onkologie, Psychosomatik und Sexualmedizin an der Versorgung andrologischer Patienten beteiligt. Die entsprechenden Kooperationspartner sind an einer klinischen Einrichtung eher als in einer Einzelpraxis unmittelbar verfügbar und können über die konsiliarische Zusammenarbeit hinaus Patienten bzw. Paare in gemeinsamen Sprechstunden betreuen (s. Abb. 1).

\subsection{Diagnostische Möglichkeiten in der klinischen Andrologie}

Die andrologische Diagnostik umfasst die ausführliche Erhebung der allgemeinen und speziellen Anamnese, die körperliche Untersuchung, Sonografie, ausgewählte Hormonanalysen; bei Fertilitätsstörungen stellt die Ejakulatanalyse ein zentrales Element dar. Gegebenenfalls sind Zusatzuntersuchungen wie z.B. humangenetische 


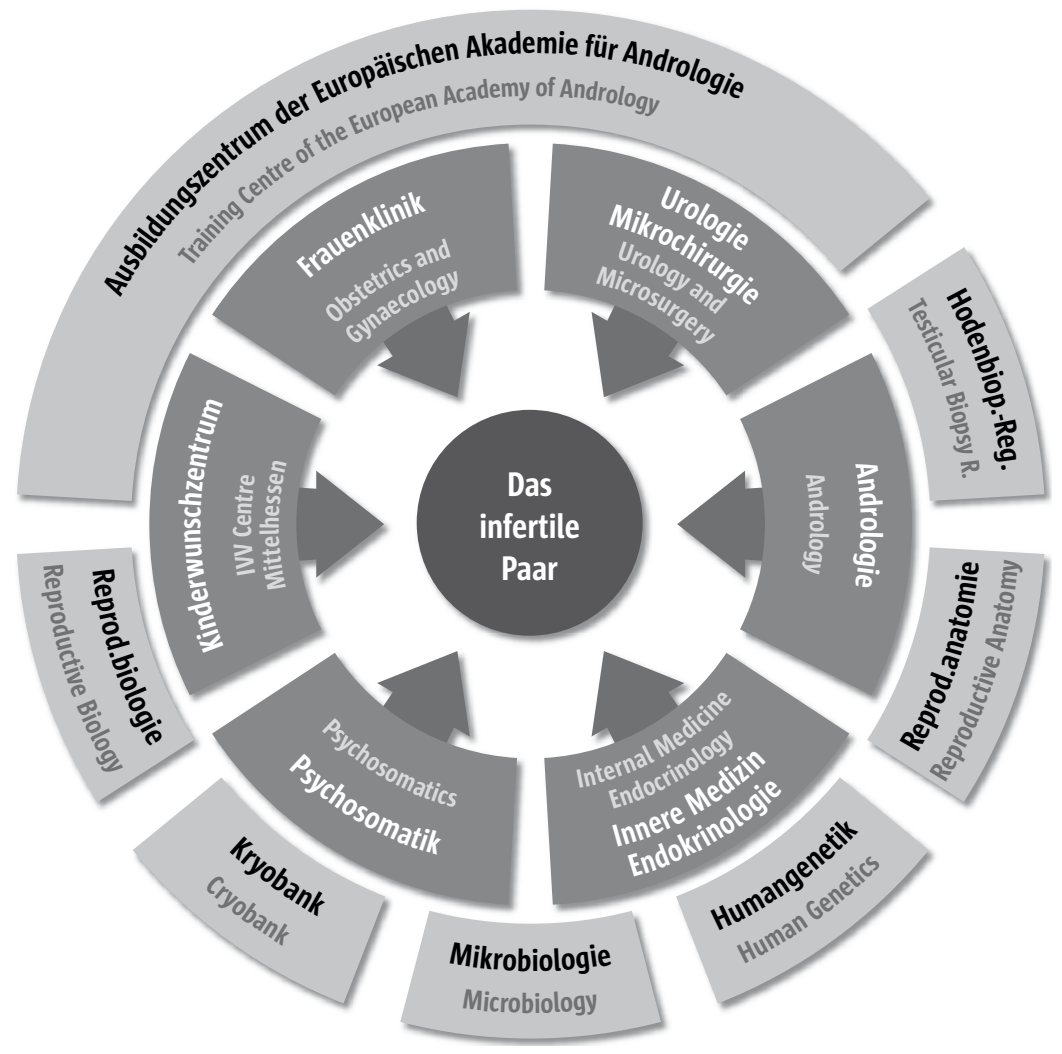

Abb. 1 Interdisziplinäre Vernetzung eines universitären andrologischen Zentrums am Beispiel des Hessischen Zentrums für Reproduktionsmedizin, Justus-Liebig-Universität Gießen (৫ Hessisches Zentrum für Reproduktionsmedizin, http://www.med.uni-giessen.de/hzrm)

Diagnostik oder Hodenbiopsie, aber auch weiterführende bildgebende Verfahren und Funktionstests indiziert (s. Kap. II.1; II.6; II.11).

\subsubsection{Apparative Untersuchungsmethoden}

\section{Sonografie}

Durch die Sonografie sind Skrotalinhalt, Prostata und Bläschendrüsen darstellbar. Die Skrotalsonografie sollte zum Standardprogramm jeder andrologischen (Erst-) Untersuchung gehören, insbesondere zur Entdeckung malignitätsverdächtiger Läsionen (Hodentumoren). Darüber hinaus können Zysten und Mikroverkalkungen (sog. „Schneegestöbermuster“) des Hodens oder auch Hydrozelen identifiziert werden, ebenso erlaubt die Hodensonografie eine präzise Bestimmung des Hodenvolumens. Am Nebenhoden imponiert eine Spermatozele als reflexfreie, glatt begrenzte runde Struktur; chronisch-entzündliche Prozesse führen neben einer Verbreiterung des Nebenhodens aufgrund narbiger Veränderungen zu echoreichen Strukturen mit unregelmäßiger Begrenzung. 
Die transrektale Sonografie lässt Veränderungen an der Prostata bei Prostatitis sowie der benignen Prostatahyperplasie erkennen und ist Bestandteil der Diagnostik eines Prostatakarzinoms. Darüber hinaus können intraprostatische Zysten und Dilatationen des Ductus ejaculatorius erfasst werden, was insbesondere bei Samentransportstörungen bzw. zentralen Verschlüssen der Samenwege von Bedeutung ist (kennzeichnend sind Azoospermie, niedriges Ejakulatvolumen, erniedrigte Fruktose und $\alpha$-Glukosidase im Ejakulat, pH-Verschiebungen in den sauren Bereich). Darüber hinaus ermöglicht die transrektale Sonografie die genaue Volumenbestimmung der Prostata.

Die Bläschendrüsen sollten vor einer evtl. Ejakulatabgabe untersucht werden. Pathologische Befunde hier können eine Aplasie oder Hypoplasie sein (z.B. bei kongenitaler bilateraler Aplasie der ableitenden Samenwege; CBAVD), auch zystische Dilatationen sind möglich.

\section{Dopplersonografie (Duplex-, Farbduplexsonografie)}

Für die Varikozelendiagnostik ist die Erfassung der Strömungsverhältnisse im Plexus pampiniformis bei Valsalva-Verfahren von Bedeutung. Hierbei wird ein venöser Reflux hörbar und durch die farbkodierte Duplexsonografie sichtbar gemacht.

Darüber hinaus kommt im Rahmen der Diagnostik der erektilen Dysfunktion die dopplersonografische Ableitung der proximalen und distalen Pulse der Aa. profundae penis und dorsales penis zur Anwendung, am besten nach intrakavernöser Verabreichung vasoaktiver Substanzen wie z.B. Prostaglandin E1 (Pharmako-Duplexsonografie). Auch ein sog. venöses Leck kann mit diesem Verfahren erfasst werden. Zur weiterführenden Abklärung einer erektilen Dysfunktion kann die Messung nächtlicher peniler Tumeszenzen durch spezielle Messgeräte (z.B. Rigiscan) eingesetzt werden, (Pharmako-)Kavernosografie oder selektive Penisangiografie sind selten notwendig.

\section{Thermografie}

Als orientierende Untersuchung bei der Varikozelendiagnostik kann die Kontaktthermografie durch Anzeigen einer hyperthermen Zone eingesetzt werden. Die Thermografie kann durch thermosensible Folien oder kontinuierlich über 24 Stunden mittels eines tragbaren Messgeräts mit Thermofühler vorgenommen werden. In der andrologischen Routine wird die Thermografie allerdings nicht regelmäßig durchgeführt.

\section{Weitere apparative Untersuchungsmethoden}

Bei urologischen Fragestellungen (z.B. mechanische oder funktionelle Blasenentleerungsstörungen) kommen urodynamische Verfahren (Uroflowmetrie) zum Einsatz. Bei Verdacht auf Strikturen und Anomalien in der Harnröhre (Divertikel oder auch Fremdkörper) wird eine Urethrozystoskopie durchgeführt.

Im Rahmen weiterführender Untersuchungen bei sekundärem Hypogonadismus mit Verdacht auf pathologische Prozesse im Bereich der Hypophyse oder des Hypothalamus ist die Magnetresonanztomografie (MRT) die Methode der Wahl.

Zur Knochendichtemessung bei Patienten mit Androgenmangel wird die sog. DEXAScan Methode (Dual energy X-ray absorptiometry) durchgeführt. 


\subsubsection{Ejakulatdiagnostik im andrologischen Labor}

Die Ejakulatanalyse ist zentraler Bestandteil der andrologischen Diagnostik und umfasst die makroskopische und mikroskopische Spermaanalyse, biochemische, immunologische und mikrobiologische Untersuchungen sowie ggf. die Durchführung spezieller Spermienfunktionstests (s. Kap. II.1). Zur Standardisierung der Ejakulatanalyse hat die WHO ein Laborhandbuch herausgegeben, das mittlerweile in der 5. englischsprachigen Auflage zur Verfügung steht (World Health Organization 2010). Aufgrund des hohen Zeitaufwands und der erforderlichen apparativen Ausstattung ist in der Praxis zumeist nur eine basale Ejakulatdiagnostik („Basis-Spermiogramm“) möglich, weiterführende Untersuchungen werden dagegen in spezialisierten Zentren vorgehalten.

Die erweiterte zytomorphologische Differenzierung des Ejakulats erfordert aufwändige Färbungen von Ausstrichpräparaten (z.B. modifiziert nach Papanicolaou oder Shorr; World Health Organization 2010), hinzukommen immunzytochemische Verfahren zur Identifizierung und Quantifizierung von Leukozyten-Subpopulationen im Rahmen einer speziellen Entzündungsdiagnostik. Chronische Entzündungen des männlichen Genitaltraktes verlaufen häufig asymptomatisch, ohne signifikante Erhöhung der Anzahl Peroxidase-positiver Leukozyten im Ejakulat (Parameter des „Basis-Spermiogramms“). Aus diesem Grund ist die zusätzliche Bestimmung von Entzündungsmarkern wie der Granulozyten-Elastase oder pro-inflammatorischer Zytokine wie z.B. Interleukin 6 oder 8 im Ejakulat sinnvoll (Kopa et al. 2005; Ostad et al. 1998). Die Messung von reaktiven Sauerstoffspezies im Ejakulat mittels Chemilumineszenzverfahren kommt dagegen zumeist nur im experimentellen Umfeld zur Anwendung. $\mathrm{Zu}$ den einfachen, auch in der Praxis realisierbaren Tests zählt der Nachweis von Spermien-Antikörpern mittels MAR-Test bzw. Immunobead-Test.

Biochemische Marker im Ejakulat werden zur Beurteilung der sekretorischen Funktion der akzessorischen Drüsen im männlichen Reproduktionstrakt sowie der entsprechenden Differenzialdiagnostik bei Samentransportstörungen bzw. Verschlüssen der Samenwege herangezogen (Fruktose: Bläschendrüsen; saure Phosphatase, Zink, PSA: Prostata; $\alpha$-Glukosidase: Nebenhoden; s. Kap. II.1). Die entsprechenden Analysen im Ejakulat bzw. Seminalplasma werden in den Labors andrologischer Zentren vor Ort durchgeführt.

In der andrologisch-gynäkologischen Zusammenarbeit, insbesondere bei der Indikationsstellung für Maßnahmen der assistierten Reproduktion und hier der Frage nach dem Fertilisierungspotenzial der männlichen Gameten, spielen Spermienfunktionstests eine wichtige Rolle (s. Kap. II.1). Entsprechender Klärungsbedarf besteht nicht zuletzt bei einer ausbleibenden Fertilisierung der Oozyten nach In-vitro-Fertilisation (IVF) bzw. Intrazytoplasmatischer Spermieninjektion (ICSI).

Die Durchführung des Basis-Spermiogramms ist zwar ein essenzieller Bestandteil der andrologischen Diagnostik, Aussagen über den Fertilitätsstatus sind hierdurch jedoch nur bedingt möglich. Zur Charakterisierung wichtiger Spermieneigenschaften und -funktionen wurden Tests wie die Bestimmung der Akrosinaktivität und der Akrosomreaktion, der humane Zona pellucida-Bindungstest und die Chromatinkondensation sowie der Hamster-Ei-Penetrationstest entwickelt. In letzter Zeit haben vor allem Tests zur Erfassung der DNA-Integrität der Spermien, und damit letztlich der frühzeitigen Apoptose, vermehrte Aufmerksamkeit erlangt, da eine Assoziation zwi- 
schen verminderten Schwangerschaftsraten nach IVF bzw. ICSI und einer gestörten DNA-Integrität beschrieben wurde. Nicht zuletzt soll auch eine erhöhte Rate von wiederholten Frühaborten mit einer gestörten DNA-Integrität der Spermien zusammenhängen (Bellver et al. 2010). Die DNA-Integrität wird z.B. mittels des sperm chromatin structure assays (SCSA) untersucht. Eine ähnliche Aussage wird durch den TUNELoder den Comet-Assay ermöglicht (Chan et al. 2001; Evenson u. Jost 1994; Huang et al. 2005; Sun et al. 1997). Die prädiktive Bedeutung dieser Tests befindet sich jedoch noch in der Diskussion, ebenso steht eine allgemeingültige Validierung aus (Paasch et al. 2007). Deshalb sollten diese neuen Untersuchungsverfahren zunächst nur an spezialisierten Zentren durchgeführt und in klinischen Studien weiter bewertet werden.

\subsection{Therapeutische Möglichkeiten}

Als Therapieansätze in der Andrologie, hier insbesondere der Fertilitätsstörungen des Mannes, bieten sich an:

1. Vermeidung schädlicher Einflüsse (z.B. exogene Noxen)

2. intensive Beratung

3. medikamentöse Behandlung (kausal/empirisch)

4. operative Maßnahmen

5. Methoden der assistierten Reproduktion

Während die Optionen 1 bis 3 ohne Weiteres in der Praxis umgesetzt werden können, sind die operativen Behandlungen einschließlich der operativen Spermiengewinnung für eine assistierte Fertilisation (ICSI) eher eine Domäne der Andrologie in der Klinik. Die operative Spermiengewinnung erfolgt naturgemäß in enger Kooperation mit reproduktionsmedizinischen Zentren (s. Kap. II.1).

\subsubsection{Operative Andrologie}

Operative Maßnahmen in der Andrologie umfassen Refertilisierungsoperationen wie Vasovasostomie und Epididymovasostomie, die Beseitigung einer Varikozele durch hohe Ligatur oder mikrochirurgische Intervention. Die Varikozelenbehandlung kann auch mit semi-invasiven Verfahren wie der retrograden oder anterograden Sklerosierung vorgenommen werden. Ein weiteres Verfahren, wenn auch selten zum Einsatz kommend, ist die Beseitigung zentraler Verschlüsse, z.B. einer Utriculuszyste. Eine Hodenbiopsie allein zu diagnostischen Zwecken ist nur selten indiziert (s.u.). Im weiteren Sinne ist auch die operative Korrektur von Fehlbildungen oder Erkrankungen im Bereich des Penis, die mit Störungen der Samendeposition bzw. einer Beeinträchtigung der Kohabitationsfähigkeit einhergehen, zu nennen.

\section{Operative Spermiengewinnung}

Die operativen Eingriffe im Zusammenhang mit einer assistierten Fertilisation(ICSI), d.h. zur Spermiengewinnung, umfassen im Wesentlichen zwei Verfahren.

- Bei Patienten mit weitgehend ungestörter Spermatogenese und Obstruktionen im Bereich der ableitenden Samenwege oder medikamentös nicht einstellbaren Spermientransportstörungen kann durch Aspiration von Spermien aus 
dem Nebenhoden (mikrochirurgische epididymale Spermienaspiration, MESA) in der Regel eine ausreichende Menge von beweglichen Spermien gewonnen werden (Schroeder-Printzen et al. 2000).

- Für Patienten mit nicht-obstruktiver Azoospermie oder eine selbst für die ICSI zu geringe Spermienzahl im Ejakulat steht die operative Hodengewebsentnahme mit nachfolgender Isolierung und Kryokonservierung testikulärer Spermien (testikuläre Spermienextraktion, TESE) für eine spätere ICSI zur Verfügung. Bei Patienten mit besonders deutlich eingeschränkter Spermatogenese kann auch eine mikroskopisch assistierte TESE (Mikro-TESE) durchgeführt werden, mit dem Ziel der Identifizierung und gezielten Entnahme von Hodengewebe aus Arealen mit potentiell erhaltener Spermatogenese (Diemer et al. 2011).

\section{Elektrostimulation}

Bei Patienten mit Querschnittlähmung kann versucht werden, mittels rektaler Elektrostimulation eine Ejakulation herbeizuführen. Da jedoch bei den meisten Patienten hierzu eine Vollnarkose erforderlich ist und sehr häufig eine äußerst schlechte Spermienqualität vorgefunden wird, ist heutzutage für solche Patienten eher eine TESE zu empfehlen.

\section{Hodenbiopsie}

Während Hodenbiopsien in früheren Jahren vor allem bei einer Diskrepanz zwischen klinischen, endokrinologischen und spermatologischen Befunden, zur Diagnosesicherung sowie Klärung möglicher Therapieoptionen bei Fertilitätsstörungen durchgeführt wurden, steht heute die Gewinnung von Gewebsproben für die TESE im Vordergrund.

Bei 40-6o\% der Patienten mit nicht-obstruktiver Azoospermie, d.h. einem primär testikulären Schaden und zumeist erhöhtem Serum-FSH, lassen sich fokal Samenkanälchen mit erhaltener Spermatogeneseaktivität und damit reife elongierte Spermatiden bzw. Spermien für eine ICSI-Behandlung finden (Schulze et al. 1999; Schwarzer et al. 2003; Pantke et al. 2008).

Hodenbiopsien können in Lokal-, Regional- oder Allgemeinanästhesie über eine begrenzte Inzision von Skrotalhaut und äußeren Hodenhüllen („Knopflochbiopsie“) oder als explorativer Eingriff mit vollständiger Freilegung von Hoden und Nebenhoden durchgeführt werden; letzteres ist bei Durchführung einer Mikro-TESE unerlässlich. Zur Erfassung seitendifferenter Befunde sollten grundsätzlich beide Hoden biopsiert werden, für eine erfolgreiche TESE ist jeweils die Entnahme mehrerer Gewebsproben an verschiedenen Stellen unter Berücksichtigung des Gefäßverlaufs zu empfehlen (Diemer et al. 2011). Sowohl im Hinblick auf die Histopathologie als auch auf die Ergebnisse der Spermienisolierung sind offene Biopsien der perkutanen Aspiration durch blinde Punktion des Hodens überlegen (Ezeh et al. 1998; Rosenlund et al. 1998).

Die Durchführung von Hodenbiopsien sollte heute grundsätzlich mit der Möglichkeit einer Kryokonservierung von Hodengewebe oder extrahierter Spermien für spätere Maßnahmen der assistierten Fertilisation verbunden sein (Baukloh 2002; Schwarzer et al. 2003). 


\subsubsection{Methoden der assistierten Reproduktion (ART)}

Sind konservative und ggf. operative Maßnahmen zur Verbesserung der Ejakulatqualität bzw. Fertilität ausgeschöpft, kommen verschiedene Methoden der ART zur Behandlung des unerfüllten Kinderwunsches infolge männlicher Fertilitätsstörungen in Betracht (s. Kap. II.1). ART zielen zunächst darauf ab, die Rahmenbedingungen einer natürlichen Fertilisation zu verbessern. Dies gilt für die intrauterine Insemination (IUI) oder die konventionelle IVF. Bei schweren Fertilitätsstörungen des Mannes verbleibt lediglich die Option einer ICSI.

\section{Fazit}

Die Andrologie als ausgesprochen interdisziplinäres Fach kann ihre Vielfalt besonders in klinischen Einrichtungen entfalten (s. Abb. 1). Dem tragen auch die Europäische Akademie für Andrologie (EAA) sowie die Europäischen urologischen Andrologen (ESAU) Rechnung, wobei nach entsprechender Zertifizierung die Anerkennung als Ausbildungs- und Trainingszentrum nach den Richtlinien der EAA erworben werden kann. In Deutschland existieren 4 EAA-Zentren.

\section{Literatur}

Baukloh V (2002) Retrospective multicentre study on mechanical and enzymatic preparation of fresh and cryopreserved testicular biopsies. Hum Reprod17, 1788-1794

Bellver I, Meseguer M, Muriel L, Garcia-Herrero S, Barreto MA, Garda AL, Remohi J, Pellicer A, Garrido N (2010) Y chromosome microdeletions, sperm DNA fragmentation and sperm oxidative stress as causes of recurrent spontaneous abortion of unknown etiology. Hum Reprod 25, 1713-1721

Chan PI, Corselli JU, Patton WC, Jacobson JD, Chana SR, King A (2001) A simple comet assay for archived sperm correlates DNA fragmentation to reduced hyperactivation and penetration of zona-free hamster oocytes. Fertil Steril 75, 186-192

Coetzee K, Kruge TF, Lombard Cl (1998) Predictive value of normal sperm morphology: a structured literature review. Hum Reprod Update 4, 73-82

Diemer T, Hauptmann A, Weidner W (2011) Therapie der Azoospermie. Operative Spermiengewinnung (MESA, TESE, Mikro-TESE). Urologe A 50, 38-46

Evenson D, Jost L (1994) Sperm chromatin structure assay: DNA denaturability. Methods Cell Biol 42 Pt B, 159-176

Ezeh UI, Moore HD, Cooke ID (1998) A prospective study of multiple needle biopsies versus a single open biopsy for testicular sperm extraction in men with non-obstructive azoospermia. Hum Reprod 13, 3075-3080

Huang CC, Lin DP-C, Tsao HM, Cheng TC, Liu CH, Lee MS (2005) Sperm DNA fragmentation negatively correlates with velocity and fertilization rates but might not affect pregnancy rates. Fertil Steril 84, 130-140

Kopa Z, Wenzel I, Papp GK, Haidl G (2005) Role of granulocyte elastase and interleukin-6 in the diagnosis of male genital tract inflammation. Andrologia 37, 188-194

Okada H, Dobashi M, Yamazaki T, Hara I, Fujisawa M, Arakawa S, Kamidono S (2002) Conventional versus microdissection testicular sperm extraction for nonobstructive azoospermia. J Urol 168, 1063-1067

Ostad M, Liotta D, Ye Z, Schlegel PN (1998) Testicular sperm extraction for nonobstructive azoospermia: results of a multibiopsy approach with optimized tissue dispersion. Urology 52, 692-696

Paasch U, Grunewald S, Glander HJ (2007) DNA-Schäden ejakulierter Spermatozoen: ein prädiktiver Faktor der männlichen Infertilität. / Reproduktonsmed Endokrinol 4, 124-139

Pantke P, Diemer T, Marconi M, Bergmann M, Steger K, Schuppe H-C, Weidner W (2008) Testicular sperm retrieval in azoospermic men. Eur Urol Suppl 7, 703-714

Rosenlund B, Kvist U, Ploen L, Rozell BL, Sjoblom P, Hillensjo T (1998) A comparison between open and percutaneous needle biopsies in men with azoospermia. Hum Reprod 13, 1266-1271 
Schlegel PN (1999) Testicular sperm extraction: microdissection improves sperm yield with minimal tissue excision. Hum Reprod 14, 131-135

Schroeder-Printzen I, Zumbé I, Bispink L, Palm S, Schneider U, Engelmann U, Weidner W (2000) Microsurgical epididymal sperm aspiration: aspirate analysis and straws available after cryopreservation in patients with non-reconstructable obstructive azoospermia. MESA/TESE Group Giessen. Hum Reprod 15, 2531-2535

Schulze W, Thoms F, Knuth UA (1999) Testicular sperm extraction: comprehensive analysis with simultaneously performed histology in 1418 biopsies from 766 subfertile men. Hum Reprod 14 Suppl 1, 82-96

Schwarzer JU, Fiedler K, Hertwig I, Krusmann G, Würfel W, Muhlen B, Pickl U, Lochner-Ernst D, Schleyer M, OvensRader A, Hennig M (2003) Male factors determining the outcome of intracytoplasmic sperm injection with epididymal and testicular spermatozoa. Andrologia 35, 220-226

Silber SI (2000) Microsurgical TESE and the distribution of spermatogenesis in non-obstructive azoospermia. Hum Reprod 15, 2278-2284

Sun JG, Jurisicova A, Casper RF (1997) Detection of deoxyribonucleic acid fragmentation in human sperm: correlation with fertilization in vitro. Biol Reprod 56, 602-607

World Health Organization (2010) WHO laboratory manual for the examination and processing of human semen 5th edition 